International Journal of Theoretical Physics (IJTP) DOI 10.1007/s10773-007-9359-5
The original publication is available at www.springer.com

Int.J.Theor.Phys. 46

\title{
Horizon-less Spherically Symmetric Vacuum-Solutions in a Higgs Scalar-Tensor Theory of Gravity
}

\author{
Nils M. Bezares-Roder ${ }^{1 *}$, Hemwati Nandan ${ }^{\dagger \dagger}$ and Heinz Dehnen ${ }^{3 \ddagger}$ \\ 1. Institut für Theoretische Physik, Universität Ulm, \\ Albert-Einstein-Allee 11,D-89069 Ulm, Germany. \\ 2. Centre for Theoretical Studies, Indian Institute of Technology, 721302 Kharagpur, India. \\ 3. Fachbereich Physik, Universität Konstanz, Postfach M677, 78457 Konstanz, Germany.
}

\begin{abstract}
The exact static and spherically symmetric solutions of the vacuum field equations for a Higgs Scalar-Tensor theory (HSTT) are derived in Schwarzschild coordinates. It is shown that in general there exists no Schwarzschild horizon and that the fields are only singular (as naked singularity) at the center (i.e. for the case of a pont-particle). However, the Schwarzschild solution as in usual general relativity (GR) is obtained for the vanishing limit of Higgs field excitations.
\end{abstract}

PACS numbers: 14.80. Bn; 12.30Dm; 11.30 Pb.; 11.15.Kc.; 98.80.Cq.

Keywords: Higgs scalar tensor theory, gravity, Schwarzschild horizon, singularity and black holes.

The standard model (SM) of elementary particle physics has been remarkably successful in providing the astonishing synthesis of the electromagnetic, weak and strong interactions of fundamental particles in nature [1, 2], and according to it, any massive elementary particle is, in fact, surrounded by a scalar meson cloud represented by the excited scalar Higgs field that acts as a source of the mass of the particle. The Higgs mechanism [3, 4] , therefore, provides a way of the acquisition of mass by the gauge bosons and fermions in nature. In view of these developments, the gravitational field equations with an unknown additional, ad hoc minimally coupled scalar field added as source in the Hilbert-Einstein field equations was examined in detail in the past [5], assuming for simplicity the scalar field as massless; it was shown that any such scalar field influences and modifies the metric independently from its strength in such a way that there exists always a simultaneous solution of the massless scalar field equation and Einstein's field equations for the static case with a scalar point-charge as a source. In any case no Schwarzschild horizon appears and only at the pointparticle the metric and the scalar field result singular as naked singularity; this is a similar situation to that of the Reissner-Nordström solution [6], only in a more general way, since singularities are avoided there only in the case of sufficiently strong electric charges compared with the mass.

General relativistic models with a scalar field, which is coupled to the tensor field of GR, are conform equivalent with more-dimensional general relativistic models [7]. Thus, following the isomorphy theorem [8], projective spaces as Kaluza-Klein's can be reduced to usual Riemannian 4-spaces, whereas a functional 5th component of the projective metric plays the role of a variable

\footnotetext{
*e-mail: Nils.Bezares@uni-ulm.de

†e-mail: hntheory@yahoo.co.in

${ }^{\ddagger}$ e-mail: Heinz.Dehnen @uni-konstanz.de
}

gravitational "constant" (as first worked out in [9]), by which it is possible to vary the strength of gravitation [10] in scalar-tensor theories. Following this and willing to get a Lagrangian-based model from which the characteristics of the scalar field can be known, in the present paper, an analogous approach as the one in 5] is fulfilled, but for the case of a non-minimally coupled Higgs field within the Higgs Scalar-Tensor theory (HSTT); it is shown that this can lead to regular fields except for the point-particle as naked singularity.

The interaction of the Higgs field with the particles that acquire mass is of short-ranged gravitational type 11, 12, 13] and is also compatible with Dirac's large number hypothesis [14] and with Einstein's Mach-Principle 15], with mass appearing through gravitational interaction. Utilizing the Jordan-Brans-Dicke theory [8, 9] and Zee's ideas of induced gravity [16], such Higgs gravitation was first acquainted by Dehnen and Frommert [17, 18], coupling the Higgs field $\phi$ non-minimally with the curvature scalar $R=g^{\mu \nu} R_{\mu \nu}$ of General Relativity (GR) and combining gravity with symmetry breakdown. Such theories have been subsequently used to explain various diverse physical phenomena viz. dark matter and flat rotation curves of spiral galaxies 19, 20, 21], quintessence and cosmological inflation [22, 23, 24].

Let us consider the uniquely formed Lagrangian in the natural system of units [17] as follows,

$$
\mathcal{L}=\left[\frac{\check{\alpha}}{16 \pi} \phi^{\dagger} \phi R+\frac{1}{2} \phi_{; \mu}^{\dagger} \phi^{; \mu}-V(\phi)\right] \sqrt{-g}+\mathcal{L}_{M} \sqrt{-g}
$$

where $\check{\alpha}$ is a dimensionless parameter and $\mathcal{L}_{M} \sqrt{-g}$ is the Lagrangian for the fermionic and massless bosonic fields. The Higgs potential in Eq.(11), which is normalized in such a way that $V\left(\phi_{0}\right)=0$ is valid for the ground state value $\phi=\phi_{0}$ of the scalar field, yields

$$
V(\phi)=\frac{\mu^{2}}{2} \phi^{\dagger} \phi+\frac{\lambda}{24}\left(\phi^{\dagger} \phi\right)^{2}+\frac{3}{2} \frac{\mu^{4}}{\lambda}=\frac{\lambda}{24}\left(\phi \phi^{\dagger}+6 \frac{\mu^{2}}{\lambda}\right)^{2},
$$


with $\mu^{2}<0$ and $\lambda>0$ as real-valued constants.

The Higgs field in the spontaneously broken phase of symmetry leads to the ground state value of the Higgs field as

$$
\phi_{0} \phi_{0}^{\dagger}=v^{2}=-\frac{6 \mu^{2}}{\lambda},
$$

which can further be resolved as $\phi_{0}=v N$ with $N=$ const. satisfying $N^{\dagger} N=1$. With the introduction of that unitary gauge [13, 21], the general Higgs field $\phi$ may then be written in terms of the real-valued excited Higgs scalar field $\xi$ in the following form:

$$
\phi=v \sqrt{1+\xi} N .
$$

The length scale of this Higgs field is given through

$$
l=\left[\frac{1+\frac{4 \pi}{3 \ddot{\alpha}}}{16 \pi G\left(\mu^{4} / \lambda\right)}\right]^{1 / 2}=M^{-1}
$$

with the Higgs field mass $M$, while the gravitational coupling parameter $G$ is then defined through the ground state value of the Higgs field given by Eq.(3):

$$
G=\frac{1}{\breve{\alpha} v^{2}}=-\frac{1}{\breve{\alpha}} \frac{\lambda}{6 \mu^{2}} .
$$

The dimensionless parameter $\breve{\alpha}$ in Eq.(1) may be defined in terms of the ratio

$$
\breve{\alpha} \simeq\left(M_{P} / M_{W}\right)^{2} \gg 1,
$$

where $M_{P}$ and $M_{W}$ are the Planck mass and the mass of the gauge boson, respectively. The mass of the gauge boson is given through

$$
M_{W}=\sqrt{\pi} g v,
$$

where $g$ is the coupling constant of the corresponding gauge group. The effective gravitational coupling, however, is given through

$$
G_{e f f}=G(\xi)=(1+\xi)^{-1} G,
$$

which reduces to Eq. (6) in the absence of the excited Higgs scalar field $\xi$ (i.e. for $\xi=0$ ) and gets singular for a vanishing Higgs scalar field with $\xi=-1$ [21].

The coupling (given through $\mathcal{L}_{M}$ ) of the Higgs particles to their source is only weak (i.e. $\sim G$ ) [17, 21, 25], and the Higgs field equation takes the form

$$
\xi_{; \mu}^{, \mu}+\frac{\xi}{l^{2}}=\frac{8 \pi G}{3} T,
$$

for the first of these cases, wherein $T$ is the trace of the symmetric energy-momentum tensor $T_{\mu \nu}$ belonging to $\mathcal{L}_{M} \sqrt{-g}$ in the Lagrangian given by Eq.(11), which satisfies the conservation law $T_{\mu}{ }^{\nu}{ }_{; \nu}=0$ in the here analized case where $\phi$ does not couple to the fermionic state $\psi$ in $\mathcal{L}_{M} \sqrt{-g}$. However, a coupling to SM, which means the production of the fermionic mass through this Higgs field, breaks the conservation law through a new "Higgs force" and implies simultaneously that the right hand-side of Eq.(10) vanishes identically [18, 25]. Nevertheless, this second case will be discussed later and not further here. Further, the Hilbert-Einstein equations, which reduce to the usual ones of GR for vanishing excitations $\xi$, are now given through

$$
R_{\mu \nu}-\frac{1}{2 l^{2}}(1+\xi)^{-1}\left[\xi\left(1+\frac{3}{2} \xi\right) g_{\mu \nu}+\xi_{, \mu ; \nu}\right]=-8 \pi G_{e f f}\left(T_{\mu \nu}-\frac{1}{3} T g_{\mu \nu}\right),
$$

for which the value of the Ricci curvature $R$ has already been introduced.

It is important to notice that in view of the structure of $l$ in the HSTT, only large values of the length scale $l$ are expected. Indeed, only such values within the HSTT lead to the correct explanation of flattened rotation curves of spiral galaxies without assuming dark matter [19, 21], as a consequence of long-range changes of the dynamics because of Higgs gravitation, i.e. the gravitational interaction of massive scalar Higgs-like fields, where nonminimally coupled Higgs particles interact almost only through the gravitational channel and thus with predictions in accordance with the experiments. However, given the relatively small masses $M$, it is important to notice that the limiting case of a vanishing Higgs field mass
(51) of the non-minimally coupled Higgs field as scalar field $(l \rightarrow \infty)$ can be understood as a double limit $\mu^{2} \rightarrow 0$ and $\lambda \rightarrow 0$, so that $\mu^{4} / \lambda=0$ and $v^{2}=\mu^{2} / \lambda=$ finite remain valid throughout. Thus, the ground state value keeps the degeneracy (remains the one of a Higgs mode and does not go through to one of a Wigner one) and the symmetry stays broken at low energies. The scalar field still changes the usual dynamics after symmetry breakdown and the excitations are in general non-vanishing. Thus, the field equations (11) do not reduce to the usual ones of GR and new changes in the dynamics can be acquainted to the scalar field and its gravitational Yukawatype interaction.

An analysis of the limit of vanishing non-minimally coupled Higgs field masses is important to give general char- 
acteristics of the dynamics within the HSTT, especially if these are expected as small. Thus, in the following, this Higgs mass will be neglected and, in order to solve the Eq.(10) with a vanishing Higgs field mass, let us write the line element in the spherical symmetry $\left(x^{\mu}=\left\{x^{0}=t, x^{1}=r, x^{2}=\vartheta, x^{3}=\varphi\right\}\right)$ as

$$
d s^{2}=e^{\nu}(d t)^{2}-e^{\lambda} d r^{2}-r^{2}\left(d \vartheta^{2}+\sin ^{2} \vartheta d \varphi^{2}\right),
$$

where $\nu$ and $\lambda$ are functions of the $r$ coordinate only. Under the assumption of a point-mass at $r=0$, the Higgs field equation given by Eq.(10) then takes the form

$$
\xi^{\prime \prime}-\frac{1}{2}\left(\lambda^{\prime}-\nu^{\prime}\right) \xi^{\prime}+\frac{2}{r} \xi^{\prime}=0,
$$

where the prime denotes the differentiation with respect to the radial coordinate $r$. The first derivative of the excited scalar field $\xi$ from Eq. (13) in the case of a pointmass (with internal structure (pressure)) at $r=0$ then reads

$$
\xi^{\prime}=\frac{A}{r^{2}} e^{w / 2}=\frac{A}{r^{2}} e^{(\lambda-u / 2)},
$$

where

$$
u:=\lambda+\nu \quad \text { and } \quad w:=\lambda-\nu
$$

are defined. However, the integration constant $A$ is given according to Eq.(10) with the signature of (12) in the limit $r \rightarrow \infty$ by

$$
A=-\frac{2}{3} G \int T \sqrt{-g} d^{3} x .
$$

With Eq.(14) along with

$$
q:=\ln (1+\xi),
$$

the non-trivial field equations associated to the Lagrangian (11) for the metric (12) (viz. 21] for the complete equations) lead in the case of a point-mass in vacuum to the following equations:

$$
\begin{gathered}
\frac{1}{2} r w^{\prime}=1-e^{(u+w) / 2}+r q^{\prime}, \\
u^{\prime}\left(1+\frac{r}{2} q^{\prime}\right)=\frac{r}{2} q^{\prime}\left(w^{\prime}-\frac{4}{r}\right), \\
\frac{1}{2}\left(u^{\prime}-w^{\prime}\right)=\frac{B}{r^{2}} e^{w / 2-q}=\frac{B}{A} q^{\prime},
\end{gathered}
$$

whereas Eq. (19) is the substraction of field equations and (20) is the total integral for $\nu^{\prime}$ with $B$ as an integration constant.

Using the value of $u^{\prime}$ given in Eq.(19), Eq.(20) leads to the following decoupled equation:

$$
w^{\prime}=-\frac{2(A+B)}{r^{2}} e^{w / 2-q}-\frac{A B}{r^{3}} e^{w-2 q} .
$$

Now, using (18) and (21) one immediately deduces

$$
e^{u / 2+q}=e^{-w / 2+q}+\frac{(2 A+B)}{r}+\frac{A B}{2 r^{2}} e^{w / 2-q},
$$

and, therefore, only the differential Eq.(21) remains to be solved. These considerations further lead to the solution of the excited Higgs field given by Eq.(14) in the following form for $B \neq 0$ :

$$
\xi=-1+e^{q}=-1+e^{\frac{A}{2 B}(u-w)} .
$$

Eq.(23) clearly shows that such excitations of the Higgs scalar field are only possible for a non-vanishing value of the integration constant $A$ given by Eq.(16). The exponential term with coefficient of amplitude and gravitational potential gives the deflection from completely vanishing scalar fields.

As boundary condition we postulate the Minkowski metric at spatial infinity. In order to determine the meaning of the integration constant $B$ we consider at first the asymptotic case $r \rightarrow \infty$ of the potentials, i.e. $|w| \ll 1$, $|u| \ll 1$. Then, we get from (21):

$$
\begin{gathered}
u=2 \frac{A}{r}+\frac{A B}{2 r^{2}}, \\
w=\frac{2(A+B)}{r}+\frac{A B}{2 r^{2}} .
\end{gathered}
$$

This results in

$$
\nu=\frac{(u-w)}{2}=-\frac{B}{r}
$$

and

$$
\lambda=\frac{(u+w)}{2}=\frac{A B}{2 r^{2}}+\frac{(2 A+B)}{r} .
$$

Consequently,

$$
B=\frac{2 \tilde{M}_{S}}{\breve{\alpha} v^{2}}=2 \tilde{M}_{S} G
$$

is valid in view of the equation of motion of the line element (12), where $\tilde{M}_{S}$ is the asymptotic $(r \rightarrow \infty)$ visible mass of the particle (and represents the Schwarzschild mass). Further, the differential Eq.(21) is an Abelian one and can be solved exactly. With the substitution

$$
e^{w / 2-q}=: r \tilde{g}(r)=: r \tilde{g}
$$

Eq.(21) acquires a much simpler form as given below,

$$
r \tilde{g}^{\prime}=\alpha \tilde{g}^{3}-K \tilde{g}^{2}-\tilde{g},
$$

whereas

$$
\begin{aligned}
K & :=2 A+B=2\left(A+G \tilde{M}_{S}\right) \text { and } \\
\alpha & :=-\frac{A B}{2}=-A \tilde{M}_{S} G .
\end{aligned}
$$


Eq.(30) can be integrated by using the method of separation of variables, which for $\alpha \neq 0$ reduces to the form given as

$$
\left|\frac{\tilde{g}^{2}}{1+K \tilde{g}-\alpha \tilde{g}^{2}}\right|\left|\frac{\sqrt{K^{2}+4 \alpha}+K-2 \alpha \tilde{g}}{\sqrt{K^{2}+4 \alpha}-K+2 \alpha \tilde{g}}\right|^{\frac{K}{\sqrt{K^{2}+4 \alpha}}}=\frac{C}{r^{2}} .
$$

The integration constant $C$ in Eq. (33) can be calculated in the Minkowskian limit [5] as

$$
C=\left(\frac{\sqrt{K^{2}+4 \alpha}+K}{\sqrt{K^{2}+4 \alpha}-K}\right)^{\frac{K}{\sqrt{K^{2}+4 \alpha}}} .
$$

Further, the constant $K$ turns out to be a generalized mass parameter and $\alpha$ itself can be interpreted as a product-charge in terms of $A$ and $B$, especially since Eq.(33) turns out to be formally the solution for a minimally coupled massless scalar field added to Einstein's field equations [5] (of course, more than formally only for small values of the $\xi$ field and thus $q \approx 0$ ). Thus, the non-minimally coupled massless Higgs field within the HSTT acts in an ananalogous way to a massless scalar field within Einstein's theory of gravity in [5]. The integration constants, however, are of different nature (since $K$ and the charge $\alpha$ are given by both the parameters of the fields), and a large length scale $l$ is expected, indeed. Furthermore, symmetry breakdown is still given, since the ground state stays degenerate and doesn't go through to the one of a Wigner mode.

In view of the Eqs.(22), (23) and (29), the metric components given by Eq.(12) and the scalar field by Eq.(14) for the case $B \neq 0$ may then be expressed in terms of $\tilde{g}$ in the following form:

$$
\begin{gathered}
e^{\nu}=\left[\frac{1}{r^{2} \tilde{g}^{2}}\left(1+K \tilde{g}-\alpha \tilde{g}^{2}\right)\right]^{B / K}, \\
e^{\lambda}=1+K \tilde{g}-\alpha \tilde{g}^{2}, \\
\xi=-1+\left[\frac{1}{r^{2} \tilde{g}^{2}}\left(1+K \tilde{g}-\alpha \tilde{g}^{2}\right)\right]^{\frac{A}{K}} .
\end{gathered}
$$

The only effective physical parameters remaining in the theory of the present model are the integration constants $A$ and $B$ defined by Eqs.(16) and (28), respectively.

Unfortunately, it is quite difficult to solve the equation (33) for $\tilde{g}$ explicitly. However, a transparent discussion of the properties of the solution is feasible in connection to [5]. For the limiting case $A=0$, i.e. for the equation of state $p=\frac{1}{3} \varrho$ (see the Eq.(16)) and $B \neq 0$ (i.e. $\alpha=0$ and $K=B$ ), using Eqs.(22) and (29), Eq. (30) can be exactly solved for $\tilde{g}$ in the following form (for $B=0$ follows $A=0$ directly and thus the Minkowski metric with a vanishing value of the excited Higgs field):

$$
\tilde{g}=\frac{1}{r}\left(1-\frac{B}{r}\right)^{-1}
$$

and thus for the potentials, using Eqs. (35) and (36):

$$
e^{\nu}=e^{-\lambda}=\left(1-\frac{B}{r}\right)
$$

This is the Schwarzschild metric, according to Eq.(12), and it appears for the limiting case $A=0$ (i.e. $\xi=0$ ) [26]. For general values of $A$, however, the qualitative course shown in [5] is valid. Moreover, high values of $A$ in (35) lead to a decrease in $\nu$ through the exponent $B / K$. In fact, both the metric and scalar field are regular everywhere with exception of $r=0$ as naked singularity. There exists no Schwarzschild horizon, which appears only for the case $A=0$ of a vanishing scalar field excitation. Black holes in the usual sense do not appear in the case $A \neq 0$. In this case, the scalar field does not lose its special characteristics and acts anti-gravitationally, canceling a singularity of curvature and metric except in the center as naked singularity. This is achieved with knowledge of the dynamical equations of the scalar field and from a Lagrangian (11) in the limiting case of massless fields. Herewith, the scalar field leads to a screening of the usual gravitational interaction (viz. quintessence and dark energy). For higher masses, this feature should be also valid, although in a weakened form.

An analysis on flat rotation curves within HSTT 21] without dark matter still leads to the possibility of the existence of highly massive cores for the galaxies. Moreover, these cores, if highly massive, seem to be necessarily even more massive than within standard dynamics. This heavy mass, however, should not be measured as such due to the characteristics of the scalar field excitation at the galactic center and to the vanishing of the effective value of the gravitational coupling. Only the effective value of mass is directly perceived (which, on the other side, is what may acquaint in the end for the solution of the dark matter problem) and the effect of the "real" central mass is highly screened by the presence of the scalar field, as would be the case of the gravitative interaction around this core.

\section{Acknowledgments}

The authors NMBR and HN are thankful to Prof. H. Dehnen and Prof. P. K. Raina for the hospitality and support during their stay at the Department of Physics at the University of Konstanz, Germany and the Centre for Theoretical Studies, Indian Institute of Technology, Kharagpur, India (under its visitor's programme) respectively. The authors would also like to thank Prof. F. Steiner and Dr. R. Aurich of the Institute of Theoretical Physics, University of Ulm, for support and useful comments.

These results were partially presented at the New Delhi IAGRG meeting, Feb. 2th, 2007. 
[1] T. D. Lee, Particle Physics and Introduction to Field Theory, Harwood Academic, New York (1981).

[2] M. E. Peskin and D. V. Schroeder, An Introduction to Quantum Field Throry, Addison Wesley Reading, Massachusetts (1995).

[3] P. W. Higgs, Phys. Rev. Lett. 12 (1964) 132.

[4] F. Engelert and R. Brout, Phys. Rev. Lett. 13 (1964) 321.

[5] A. Hardell and H. Dehnen, Gen. Rel. and Grav. 25 (11) (1993) 1165.

[6] H. Reissner, Ann. der Physik 50 (1916) 106.

[7] S. Cotsakis, gr-qc/9712046 v1 (1997).

[8] P. Jordan, Schwerkraft und Weltall, Vieweg (Braunschweig) (1955).

[9] C. H. Brans and R. H. Dicke, Phys. Rev. 124 (1961) 925.

[10] B. Fauser, gr-qc/0011015p1 (2001).

[11] H. Dehnen and H. Frommert, Int. J. Theor. Phys. 29 (4) (1990) 361.

[12] H. Dehnen and H. Frommert, Int. J. Theor. Phys. $\underline{29}(6)$ (1990) 537.

[13] H. Dehnen and H. Frommert, Int. J. Theor. Phys. $\underline{30}$ (1991) 985.

[14] C. H. Brans, "Gravity and the Tenacious Scalar Field", gr-qc/9705069 (1997).

[15] A. Einstein, Phys. Zs. 14 (1913) 1260.
[16] A. Zee, Phys. Rev. Lett. 42 (7) (1979) 417.

[17] H. Dehnen, H. Frommert and F. Ghaboussi, Int. J. Theor. Phys. 31 (1) (1992) 109.

[18] H. Dehnen, H. Frommert, Int. J. Theor. Phys. $\underline{32}$ (7) (1993) 1135.

[19] E. Gessner, Astrophys. and Sp. Sc. 196 (1992) 29.

[20] M. A. Rodríguez-Meza and J. L. Cervantes-Cota, Mon. Not. R. Astron. $\underline{350}$ (2004) 671.

[21] N. M. Bezares-Roder and H. Dehnen, Gen. Relativ. Gravit. 39(8) (2007) 1259.

[22] J. L. Cervantes-Cota and H. Dehnen, Phys. Rev. D $\underline{51}$ (1995) 395.

[23] J. L. Cervantes-Cota and H. Dehnen, Nucl. Phys. B $\underline{442}$ (1995) 391.

[24] A. L. Berkin and K. Maeda, Phys. Rev. D $\underline{44}$ (1991) 1691.

[25] N. M. Bezares Roder and H. Nandan, to appear in IJP hep-ph/0603168. (2007).

[26] N.M. Bezares-Roder, H. Nandan, H. Dehnen, Schwarzschild solution in a Higgs scalar-tensor theory of gravity. In: 24th IAGRG Meeting, New Delhi, Recent Advances in Gravitation and Cosmology (2007). 